\title{
TEACHING EXCHANGE
}

\section{Developing and piloting a resource for training assessors in use of the Mini- CEX (mini clinical evaluation exercise)}

Elaine Walsh, Tony Foley, Carol Sinnott, Siobhan Boyle, W Henry Smithson

\section{Background}

The assessment of undergraduate medical students in the clinical setting has become a key priority for medical educators. Facilitating the successful translation of undergraduate theoretical knowledge into safe and appropriate postgraduate clinical practice represents a challenge in medical education [1]. Poor clinical performance of newly qualified doctors has been highlighted as a major issue relating to patient safety [2]. Performance based assessment in the undergraduate setting may assist in addressing this issue by assessing "doing" rather than "knowing". The mini clinical evaluation exercise (Mini-CEX) is a formative assessment used to assess the performance of medical students in a clinical context. It incorporates assessment by and feedback from an assessor, based on the direct observation of a studentpatient consultation [3]. Conducted in a series of stages, the Mini-CEX allows focused assessment of key competencies (see Box 1) [3].

\section{BOX 1}

Stages of a Mini-CEX:

1. Preparation:

- Case selection

- Student instruction

2. Observation:

- Rate competencies

$>$ History taking skills

$>$ Physical examination skills

$>$ Communication

$>$ Clinical reasoning

$>$ Professionalism

$>$ Clinical judgement and decision making

$>$ Organisation and time management

- Assign overall score

3. Feedback

- Specific strengths/weaknesses

- Explore development needs

- Agree action plan 
While widely employed in both undergraduate and postgraduate settings as an assessment tool the Mini-CEX has been criticised for lacking standardisation [4,5]. Ideally, the MiniCEX assessment should be conducted on a number of occasions and assessors should receive formal training in the technique [6].

The Mini-CEX is used currently to assess third and final year undergraduate medical students in University College Cork (UCC). However, in previous work, we identified that local assessors lacked both formal training and confidence in use of the Mini-CEX [7].

Additionally, an audio-visual recording of a Mini-CEX encounter was identified by the assessors as a potentially useful resource for supporting training in use of the Mini-CEX [7]. Our aim was to develop and evaluate a training workshop for Mini-CEX assessors using an audio-visual recording of a Mini-CEX encounter with the aim of improving confidence in use of the Mini-CEX and standardising student assessment.

\section{What we did}

\section{Development of training material}

We conducted an online search for Mini-CEX training resources and also contacted both general practice and medical postgraduate training bodies to identify existing Mini-CEX training resources. A lack of suitable audio-visual material was identified. Deficits identified in the existing material included poor visual and audio quality, outdated appearance, incomplete Mini-CEX performance and lack of local relevance. To address the gap in suitable Mini-CEX training material, we convened a working group of clinical and academic staff in the Department of General Practice and staff from the Office of Media and Communications in UCC with the aim of developing a novel audio-visual recording of a Mini-CEX. Two clinical scenarios were developed to facilitate assessment of the competency of history taking. A senior and junior medical student were chosen to participate as student actors. An actress with prior experience of role-playing within an undergraduate medical teaching environment acted as the patient and was given detailed briefing on the clinical scenario. A GP acted as the assessor. Both the students and the assessor were blind to the clinical scenario. To simulate clinical practice, a general practice was chosen as the filming location. Two individual scenarios were recorded per student during a half day recording session and can be viewed at http://www.eprepp.ie/assessment/ [8].

\section{Conduct of training workshop}

Using this video material as a training resource, we developed a structured 2-hour workshop. The workshop was provided by the first and second authors (EW and TF), both GPs. As the Mini-CEX is currently used as an assessment tool in both hospital and GP placements in UCC, medical educators from multiple specialities and general practice were invited to participate in the workshop. This allowed the opportunity to gain multidisciplinary perspectives and feedback on the training material, and additionally, provided the attendees with the opportunity to exchange ideas and information regarding assessment. The structure of the workshop is outlined in Box 2. 


\section{BOX 2}

Workshop structure:

1. Introduction

2. Viewing of first Mini-CEX recording

- Marking using evaluation form

- Discussion

3. Overview of conducting a Mini-CEX

4. Viewing of second Mini-CEX recording

- Marking using evaluation form

- Discussion

Following a brief introduction, the first Mini-CEX recording was played. Attendees were asked to mark the student by ticking a box to grade the competencies of history taking, communication, clinical reasoning, professionalism, clinical judgement and organisation as fail, borderline, pass, honours or first honours, then to assign an overall grade and to provide feedback to the student. Subsequently group discussion was facilitated by EW and TF and information was provided to the attendees on the context, structure and marking of the Mini-CEX. The attendees were then shown a second Mini-CEX recording and again asked to mark the student and give feedback using the schedule provided. Written feedback was collected.

\section{Evaluation of impact of training material and workshop}

To evaluate if the workshop would increase the participants' confidence in use of the Mini-CEX, we asked each participant to rate their training in and confidence with the Mini-CEX using a Likert scale of 1-5 where 1 was "not-confident" and 5 was "very confident", prior to the workshop. After the workshop, participants were asked to repeat this rating and were also asked about their views on the usefulness of the workshop using a Likert scale where 1 was "not useful" and 5 was "very useful" and additional free-text comments. Scores before and after were compared using Mann Whitney U tests.

To evaluate if the workshop was associated with reduced variability of marks awarded for the second Mini-CEX example, marks were calculated for each assessment (Fail=1, Borderline=2, Pass=3, Honours $=4$ and First Honours=5). Median scores for the first and second Mini-CEX assessments were calculated and compared using Mann Whitney $\mathrm{U}$ tests.

Statistical analysis was conducted using SPSS (version 22). 


\section{Results and discussion}

The workshop was attended by 21 clinicians involved in undergraduate medical education: GPs ( $\mathrm{n}=$ 11), medical specialists $(n=8)$, obstetricians $(n=2)$ and surgeon $(n=1)$. Of those who attended 13 had previous experience of using the Mini-CEX as a method of student assessment but only two had received training in the use of the Mini-CEX previously.

The majority of attendees $(n=20)$ completed all sections of the marking schedule for the first MiniCEX example with one attendee not completing all sections. Fifteen of the attendees completed all sections of the marking schedule for the second Mini-CEX with 6 not completing all sections.

Regarding confidence in use of the Mini-CEX, the median confidence level was 2 pre-workshop (IQR 2 ) and was 4 post-workshop (IQR 0), a statistically significant increase $(p<0.001)$.

No difference was detected in the variability of marks for the first and second Mini-CEX examples. The median score for the first Mini-CEX example was 4 (IQR 0) and the median score for the second was 4 (IQR 1). The co-efficient of variance was 0.1259 for the first Mini CEX example and 0.2044 for the second, consistent with more variability in the marking of the second Mini-CEX. This, however, was not statistically significant $(p=0.08)$.

The majority of attendees $(n=20)$ rated the workshop as "very useful".

Free text comments identified interactive group discussion around variability/standardisation of marking, meeting with other assessors and the use of a "real" case as the most useful aspects of the workshop.

Suggested improvements to the workshop included having more detailed descriptors on marking schedules and having more time allocated for a broader discussion of assessment in general; indicating a general need for enhanced training of medical educators and assessors which has previously been reported [6].

In conclusion, the workshop increased the confidence of attendees in use of the Mini-CEX and was perceived by participants as very useful. The results support the use of an audio-visual resource in the training of assessors in use of the Mini-CEX. Additionally, the provision of Mini-CEX training in an interactive workshop setting with a multispecialty audience provided teachers of different disciplines with the opportunity to discuss general issues encountered in teaching and assessment. There was no significant change in the variability of student scores in the first and second Mini-CEX assessments. This may be accounted for by the fact that not all attendees completed the marking schedule for both videos. The range of disciplines of the assessors may also account for overall variability. However, the brevity of the workshop including the review of only one subsequent case involving a different student and a different clinical scenario may also have contributed to variability. Additionally, as both students performed well in the videos, the use of less clear-cut material may have exposed greater improvement in the variability of marking.

Our work suggests that appropriately designed Mini-CEX materials used in an interactive workshop setting may be an effective training method in Mini-CEX assessment. This simple, well-received workshop intervention warrants further larger scale evaluation regarding its impact on student performance and later clinical performance, in addition to its use as a training resource.

\section{References}


1. Kellett J, Papageorgiou A, Cavenagh P, Salter C, Miles S, Leinster SJ. The preparedness of newly qualified doctors-Views of Foundation doctors and supervisors. Medical Teach. 2015 Oct 3;37(10):949-54.

2. Dornan T, Ashcroft D, Heathfield H, Lewis P, Miles J, Taylor D, Tully M, Wass V. An in-depth investigation into causes of prescribing errors by foundation trainees in relation to their medical education: EQUIP study. London: General Medical Council. 2009 Dec:1-215.

3. Norcini JJ, Blank LL, Duffy FD, Fortna GS. The mini-CEX: a method for assessing clinical skills. Ann Intern Med. 2003 Mar 18;138(6):476-81

4. Govaerts MJ, Schuwirth LW, Van der Vleuten CP, Muijtjens AM. Workplace-based assessment: effects of rater expertise. Adv Health Sci Educ Theory Pract. 2011 May;16(2):151-65

5. McGill DA, van der Vleuten CP, Clarke MJ. Supervisor assessment of clinical and professional competence of medical trainees: a reliability study using workplace data and a focused analytical literature review. Adv Health Sci Educ Theory Pract. 2011 Aug;16(3):405-25

6. Weston PS, Smith CA. The use of mini-CEX in UK foundation training six years following its introduction: lessons still to be learned and the benefit of formal teaching regarding its utility. Med Teach. 2014 Feb;36(2):155-63

7. Foley T, Walsh E, Sweeney C, James M, Maher B, O'Flynn S. Training the assessors: A Mini-CEX workshop for GPs who assess undergraduate medical students. Education for Primary Care. 2015 Nov 2;26(6):446-7.

8. Electronic Preparation for Professional Practice (ePrePP) http://www.eprepp.ie/assessment/ 